\title{
The optical polarisation of the Vela pulsar revisited ${ }^{\star}$
}

\author{
R. P. Mignani ${ }^{1}$, S. Bagnulo ${ }^{2}$, J. Dyks ${ }^{3}$, G. Lo Curto ${ }^{2}$, and A. Słowikowska ${ }^{3,4}$ \\ 1 Mullard Space Science Laboratory, Holmbury St. Mary, Dorking - Surrey, RH5 6NT, UK \\ e-mail: rm2@mssl.ucl.ac.uk \\ 2 European Southern Observatory, Alonso de Cordova 3107, Vitacura, Santiago, Casilla 19001 Santiago 19, Chile \\ e-mail: [sbagnulo;glocurto]@eso.org \\ 3 Nicolaus Copernicus Astronomical Centre, Rabiańska 8, 87-100 Toruń, Poland \\ e-mail: [jinx; aga]@ncac.torun.pl \\ ${ }^{4}$ Max-Planck-Institut für Extraterrestrische Physik, Giessenbachstrasse, Garching, 85748 Germany \\ e-mail: aga@mpe.mpg.de
}

Received 12 July 2006 / Accepted 7 February 2007

\section{ABSTRACT}

\begin{abstract}
Aims. In this work we present a revised measurement of the phase-averaged optical polarisation of the Vela pulsar (PSR B0833-45), for which only one value has been published so far (Wagner \& Seifert 2000, ASP Conf. Ser., 202, 315).

Methods. Our measurement has been obtained through an accurate reanalysis of archival polarisation observations with the FORSI instrument at the $V L T$.

Results. We measured a phase-averaged linear polarisation degree $P_{\mathrm{L}}=9.4 \% \pm 4 \%$ and a position angle $\theta=146^{\circ} \pm 11^{\circ}$, very close to the ones for the axis of symmetry of the X-ray arcs and jets detected by Chandra and of the pulsar proper motion.

Conclusions. We compared the measured phase-averaged optical polarisation with the expectations of different pulsars' magnetosphere models. We find that all models consistently predict values of the phase-averaged linear polarisation that are too high with respect to the observed one. This is probably a consequence of present models' limitations that neglect the contributions of various depolarisation effects. Interestingly, for the outer gap model we find that, when assuming synchrotron radiation for the optical emission, the observed polarisation position angle also implies an alignment between the pulsar rotational axis and the axis of symmetry of the X-ray arcs and jets.
\end{abstract}

Key words. polarization - stars: pulsars: individual: PSR B0833-45

\section{Introduction}

Polarisation measurements of pulsars and their synchrotron nebulae are uniquely able to provide deep insights into the highly magnetised relativistic environment of young rotating neutron stars. Besides the radio band, optical observations are primarily suited to providing such insights. Being the first and the brightest $(V \sim 16.5)$ optical pulsar identified so far, it comes as no surprise that the first optical polarisation measurements were obtained for the Crab pulsar (PSR B0531+21). Strong polarisation is actually expected when the optical emission is produced by synchrotron radiation which is certified, in the case of the Crab pulsar, by its power-law spectrum (see, e.g., Sollerman et al. 2000). In fact, the polarisation of the Crab pulsar optical emission was discovered (Wampler et al. 1969) soon after its optical identification (Cocke et al. 1969). Accurate optical phase-resolved polarisation measurements of the Crab pulsar (e.g. Smith et al. 1988; Kanbach et al. 2005) have shown the phase dependence of the degree of polarisation, with a maximum of $\sim 50 \%$ in the bridge between the main and the interpulse.

Optical polarisation measurements are extremely useful in providing observational constraints to test the different models proposed for the magnetospheric activity of pulsars. Unfortunately, even though the number of pulsars detected in the optical band has increased significantly in the past ten years (see

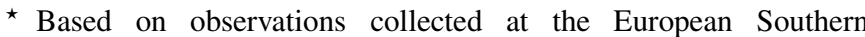
Observatory, Paranal, Chile under programme ID 63.P-0002(A).
Mignani et al. 2004 and Mignani 2005, for updated reviews), the Crab pulsar is still the only one for which both precise and repeated polarisation measurements have been obtained. For the other young pulsars (age $\leq 10000$ years) only preliminary or uncertain phase-averaged optical polarisation measurements have been reported so far.

For the second youngest $(\sim 1600$ years $)$ optical pulsar PSR B0540-69 ( $V \sim 22.5)$, considered the Crab pulsar "twin" because of its overall similarities in age, period, and energetics (e.g. Caraveo et al. 2001a), polarisation observations with the $V L T$ were reported by Wagner \& Seifert (2000). However, the polarisation measurement $(\approx 5 \%$ with no quoted error) was certainly contaminated by the contribution of the compact ( $\sim 4^{\prime \prime}$ diameter) surrounding synchrotron nebula (Caraveo et al. 2001a). For PSR B1509-58, the next youngest ( $\sim 5000$ years) pulsar with a candidate optical identification, the value of the optical polarisation is also very uncertain. The original counterpart $(V=22)$ proposed by Caraveo et al. (1994) was discarded by Wagner \& Seifert (2000) on the basis of the lack of evident optical polarisation in favour of a much fainter $(R \sim 26)$ object hidden in the PSF wings of the star, whose detection has now been confirmed by IR observations (Kaplan \& Moon 2006). However, both the difficulties in the PSF subtraction and the faintness of the counterpart make the reported polarisation measurement $(\approx 10 \%$, also quoted with no error) only tentative.

A preliminary polarisation measurement was also reported by Wagner \& Seifert (2000) for the Vela pulsar 
(PSR B0833-45), the third brightest $(V \sim 23.6)$ and youngest $(\sim 10000$ years) optical pulsar. Finally, for the older ( $\sim 100000$ years) PSR B0656+14 $(V \sim 25)$, phase-resolved optical polarisation measurements have been obtained by Kern et al. (2003). Like for the Crab, also the polarisation degree is also phase-dependent for PSR B0656+14, with a maximum $(\sim 100 \%)$ in the bridge between the two pulses. For the Vela pulsar, Wagner $\&$ Seifert (2000) only published the value of the linear polarisation degree. The need to obtain a more complete description of the polarisation properties of Vela pulsar by including, e.g. the linear polarisation position angle has prompted us to undergo a careful reanalysis of the original data set. The knowledge of the polarisation position angle is indeed an additional, important piece of information for testing different models of the optical emission of pulsars. This paper is organised as follows: observations, data analysis, and results are described in Sect. 2, while the comparison with existing pulsar emission models is discussed in Sect. 3.

\section{Observations and data analysis}

Phase-averaged polarimetry observations of the Vela pulsar were obtained at the first Unit Telescope (UT1) of the ESO Very Large Telescope with FORS1 on April 12, 1999 as a part of the instrument commissioning ${ }^{1}$. FORS 1 (FOcal Reducer Spectrograph), a multi-mode instrument for imaging and (long-slit/multi-object) spectroscopy equipped with polarimetric optics, is described in Appenzeller et al. (1998). Observations were performed in standard resolution (SR) mode ( $0.2 /$ pixel) through the $R$ Bessel filter $(\lambda=6570 \AA ; \Delta \lambda=1500 \AA)$, with an average seeing of $\sim 0{ }^{\prime} 7$ and airmass varying between 1.4 and 1.9 .

The instrument set-up was chosen to allow for linear polarisation measurements. A total of 6 frames were taken with the instrument position angle (hence the Wollaston axis) oriented at $+10^{\circ}$ North to East. One $1000 \mathrm{~s}$ plus two $380 \mathrm{~s}$ exposures were obtained with the fast axis of the half-wave retarder plate parallel to the Wollaston prims (rotation angle $0^{\circ}$ ) and the remaining three $(1000 \mathrm{~s} \mathrm{each})$ at rotation angles $22.5^{\circ}, 45^{\circ}$, and $67.5^{\circ}$, respectively (see Table 1 for a log of the observations). In this work we have used only the longest of the exposures performed with the $0^{\circ}$ retarder plate angle. Science data and the associated calibrations were retrieved through the ESO archive interface ${ }^{2}$ and reduced following the standard approach for de-biasing and flat-fielding. The master flat field was obtained from the mediancombination of sky images obtained during twilight with no polarimetric optics in the light path. No observations of polarimetric standard stars were performed during the night, but analysis of the polarimetric data taken in the context of the polarimetric calibration plan shows that the instrument is stable (Fossati et al. 2007). We finally obtained four reduced images corresponding to the four retarder plate angles. As an example, we show in Fig. 1 the reduced image taken with the $0^{\circ}$ retarder plate angle. We checked for the presence of extended polarisation features that could be spatially associated with the X-ray nebula detected around the pulsar by Chandra (Pavlov 2001), but we found none, thus confirming the quick analysis briefly reported in Mignani et al. (2003).

\footnotetext{
1 See http://archive.eso.org/archive/ eso_data_products.html

2 http://archive.eso.org/
}

Table 1. Summary of the polarimetric observations of the Vela pulsar field taken with FORS1.

\begin{tabular}{ccccc}
\hline \hline Seq. & Angle $\left({ }^{\circ}\right)$ & Exposure $(\mathrm{s})$ & Seeing $\left({ }^{\prime \prime}\right)$ & Airmass \\
\hline 1 & 0 & 1000 & 0.81 & 1.37 \\
2 & 22.5 & 1000 & 0.78 & 1.44 \\
3 & 45. & 1000 & 0.57 & 1.53 \\
4 & 67.5 & 1000 & 0.62 & 1.63 \\
5 & 0 & 380 & 0.61 & 1.76 \\
6 & 0 & 380 & 0.62 & 1.90 \\
\hline
\end{tabular}

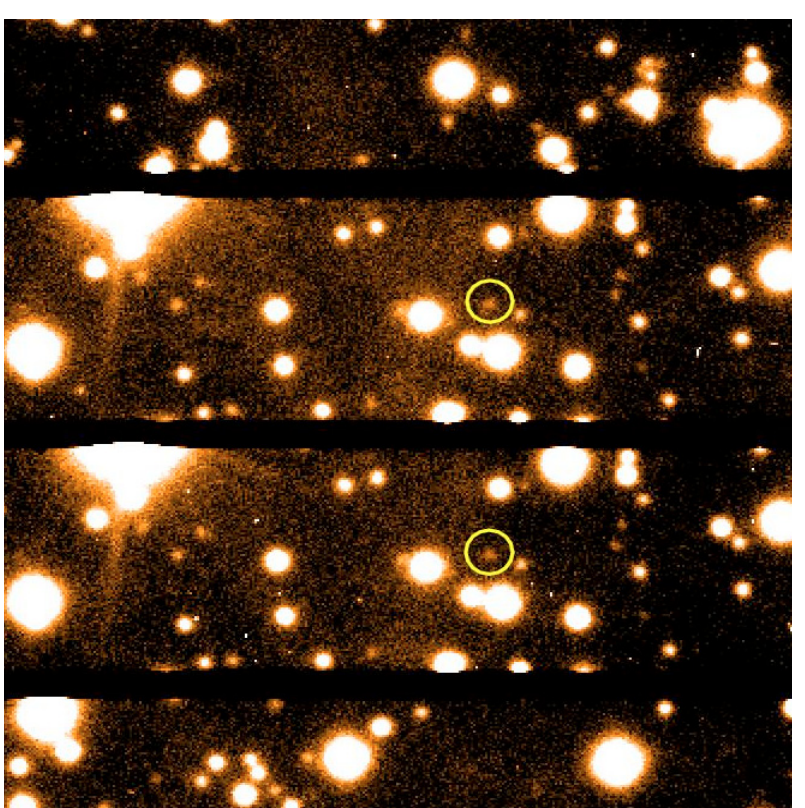

Fig. 1. VLT FORS1 image of the Vela pulsar field taken with the $R$ Bessel filter and polarised optics, which split the source image into ordinary and extra-ordinary beams. The pulsar counterpart $(R=23.9)$ is highlighted by the circle.

\subsection{Data analysis}

We then used our reduced images to compute the linear, phaseaveraged polarisation degree of the Vela pulsar. For a given source, the linear polarization degree $P_{\mathrm{L}}$ can be calculated as

$P_{\mathrm{L}}=\left(P_{\mathrm{Q}}^{2}+P_{\mathrm{U}}^{2}\right)^{1 / 2}$

where, following the notations defined in Landi Degl'Innocenti et al. (2007):

$P_{\mathrm{Q}}=\frac{Q}{I}$ and $P_{\mathrm{U}}=\frac{U}{I}$

and $I, Q, U$ are the Stokes parameters defined as in Shurcliff (1962). Applying the recipe described in Bagnulo et al. (2006), the $P_{\mathrm{Q}}$ and $P_{\mathrm{U}}$ parameters are computed according to the following relations:

$$
\begin{aligned}
& P_{\mathrm{Q}}=\frac{1}{2}\left\{\left(\frac{f^{\mathrm{o}}-f^{\mathrm{e}}}{f^{\mathrm{o}}+f^{\mathrm{e}}}\right)_{\alpha=0^{\circ}}-\left(\frac{f^{\mathrm{o}}-f^{\mathrm{e}}}{f^{\mathrm{o}}+f^{\mathrm{e}}}\right)_{\alpha=45^{\circ}}\right\} \\
& P_{\mathrm{U}}=\frac{1}{2}\left\{\left(\frac{f^{\mathrm{o}}-f^{\mathrm{e}}}{f^{\mathrm{o}}+f^{\mathrm{e}}}\right)_{\alpha=22.5^{\circ}}-\left(\frac{f^{\mathrm{o}}-f^{\mathrm{e}}}{f^{\mathrm{o}}+f^{\mathrm{e}}}\right)_{\alpha=67.5^{\circ}}\right\}
\end{aligned}
$$

where $f^{\mathrm{o}}$ and $f^{\mathrm{e}}$ are the source counts measured in the ordinary and in the extraordinary mode, respectively, for each of the four retarder plate angles $\left(0^{\circ}, 22.5^{\circ}, 45^{\circ}\right.$, and $\left.67.5^{\circ}\right)$. In our case, the 
source counts have been measured via standard aperture photometry using the photometric package of IRAF and optimised apertures for both the source and the background. For all retarder plate angles, we have obtained photometric accuracies of $\sim 8 \%$, or better. To minimise the contamination from the three brighter stars close to the pulsar position (see Fig. 1), we also measured the source counts through PSF photometry. However, the results obtained with the two approaches do not differ significantly.

By substituting the values of $f^{\mathrm{o}}$ and $f^{\mathrm{e}}$ measured for the Vela pulsar for all four retarder plate angles in Eqs. (2) and (3), we thus obtained $P_{\mathrm{Q}}=0.02 \% \pm 3.7 \%$ and $P_{\mathrm{U}}=-9.4 \% \pm 4.0 \%$, where the errors on $P_{\mathrm{Q}}$ and $P_{\mathrm{U}}$ are given by Eq. (3) of Fossati et al. (2007). These values were then corrected as explained in the FORS User Manual and in Fossati et al. (2007) to account for the chromaticity of the retarder waveplate. These values were measured in the detector reference frame, which was oriented $+10^{\circ}$ North to East (see Sect. 2.1). In order to refer them to the celestial reference frame, we applied a counter-rotation with respect to the instrument position angle, using Eq. (9) of Landi Degl'Innocenti et al. (2007) with $\chi=-10^{\circ}$. We thus end up with $P_{\mathrm{Q}}^{\prime}=3.6 \% \pm 3.7 \%$ and $P_{\mathrm{U}}^{\prime}=-8.7 \% \pm 4.0 \%$, where $P_{\mathrm{Q}}^{\prime}$ and $P_{\mathrm{U}}^{\prime}$ represent the linear polarization measured using the North Celestial Meridian as the reference direction.

\subsection{Results}

By substituting $P_{\mathrm{Q}}^{\prime}$ and $P_{\mathrm{U}}^{\prime}$ to Eq. (1), we finally obtained $P_{\mathrm{L}}=$ $9.4 \pm 4 \%$, where the associated error was calculated using Eq. (6) of Fossati et al. (2007). This relatively large error is justified by the fact that, owing to the faintness of the target $(R=23.9)$, the uncertainty on the background subtraction dominates the photometric errors on $f^{\circ}$ and $f^{\mathrm{e}}$ and ultimately those on $P_{\mathrm{Q}}^{\prime}$ and $P_{\mathrm{U}}^{\prime}$. We checked that our measurement is not affected by systematics effects such as instrumental or the background polarisation of the supernova remnant. The FORS1 instrumental polarisation has been carefully characterised by Patat \& Romaniello (2006), who find a significant spurious polarisation (at the level of $\approx 1.5 \%$ ) close to the edges of the CCD, while close to the field centre is of the order $0.03 \%$, or less. Since our target was positioned at the centre of the CCD, our measurement is not affected by the instrumental polarisation. We finally evaluated the background polarisation produced by the supernova remnant by repeating our measurements on several objects of different brightness close to the pulsar position, and we found that its effect is probably less than $1 \%$, i.e. well within our global error budget. A better characterisation of the background polarization introduced by the supernova remnants will be possible only when higher signalto-noise data are available.

While our measured polarisation degree is consistent with the one of Wagner \& Seifert (2000), who reported $P_{\mathrm{L}}=8.5 \%$, our associated error turns out to be much larger with respect to their quoted $0.8 \%$. The source of the large discrepancy in the error estimate is not clear, although we speculate that Wagner \& Seifert underestimated the error due to neglect of the background subtraction contribution to the global error budget, since $0.8 \%$ is approximately the error that we would obtain when considering the photon noise as the only source of measurement indetermination.

From the values of $P_{\mathrm{U}}^{\prime}$ and $P_{\mathrm{Q}}^{\prime}$, we computed the linear polarisation position angle $\theta$, defined as the angle from the north celestial meridian to the major axis of the polarisation ellipse
(Landi Degl'Innocenti et al. 2007). For $P_{\mathrm{Q}}^{\prime}>0$, the correct expression to compute $\theta$ is (Landi Degl'Innocenti et al. 2007)

$\theta=\frac{1}{2} \arctan \left(\frac{P_{\mathrm{U}}^{\prime}}{P_{\mathrm{Q}}^{\prime}}\right)$

where $\theta$ counted eastward from the north celestial meridian. For our values of $P_{\mathrm{U}}^{\prime}$ and $P_{\mathrm{Q}}^{\prime}$, we obtain $\theta=-34^{\circ} \pm 11^{\circ}$ or, equivalently, $\theta=146^{\circ} \pm 11^{\circ}$. The error on $\theta$ was computed using Eq. (7) of Fossati et al. (2007).

We checked for a possible coincidence between the pulsar phase-averaged optical linear polarisation position angle $\theta$ and the position angle $\theta_{\mathrm{X}}$ for the axis of symmetry of the X-ray arcs and jets detected around the pulsar by Chandra (Pavlov et al. 2001). These structures are most commonly interpreted in terms of particle outflows from the pulsar equatorial wind and of collimated outflows along the pulsar spin axis (Pavlov et al. 2001; Helfand et al. 2001). Alternatively, they are interpreted in terms of (i) particle outflows along the open field lines of the pulsar's magnetosphere and of (ii) collimated outflows along the pulsar magnetic axis (Radhakrishnan \& Deshpande 2001; Deshpande \& Radhakrishnan 2007). Different estimates exist for $\theta_{\mathrm{X}}$, e.g. $310^{\circ} \pm 1.5^{\circ}$ (Helfand et al. 2001), $307^{\circ} \pm 2^{\circ}$ (Pavlov et al. 2001), and $310.63^{\circ} \pm 0.07^{\circ}(\mathrm{Ng} \&$ Romani 2004). It has also been noted (e.g. Helfand et al. 2001) that $\theta_{\mathrm{X}}$ apparently coincides with the position angle $\theta_{\mu}$ of the pulsar proper motion. For $\theta_{\mu}$, based on their HST observations Caraveo et al. (2001b) give $307^{\circ} \pm 2^{\circ}$, while Dodson et al. (2003) give $301^{\circ} \pm 1.8^{\circ}$ with the $V L B I$. Interestingly, our measurement of $\theta$ is not very different (within $\approx 2 \sigma$ ) from the available measurements of $\theta_{\mathrm{X}}$ and $\theta_{\mu}$, suggesting an alignment between the optical linear polarisation direction, the axis of symmetry of the X-ray arcs and jets, and the pulsar proper motion. Although the chance occurrence probabilities of this alignment are not negligible, it is tantalising to speculate about it beeing a tracer of the connection between the pulsar's magnetospheric activity and its dynamical interactions with the surrounding medium. More precise measurements of the pulsar's optical polarisation, possibly supported by polarisation measurements still to come in X-rays, will hopefully provide a further observational handle for more robust theoretical speculations.

\section{Discussion}

We then compared our measurement of the phase-averaged optical polarisation degree of the Vela pulsar with the expectations of various pulsar magnetosphere models. We used the polarisation code of Dyks et al. (2004, hereafter DHR04), which can calculate the linear polarisation degree for the emission region of the outer gap model (Cheng et al. 1986; Romani \& Yadigaroglu 1995) and its two-pole version (Dyks \& Rudak 2003, hereafter DR03). The code carefully takes the macroscopic properties of the emission region into account (3D spatial extent, differential aberration, propagation delays, cumulation of radiation emitted from different parts of the emission region at the same pulse phase), but it ignores much of the micro physics. For instance, the intrinsic polarisation degree $P_{\mathrm{L}}^{\text {intr }}$ of emitted radiation is assumed to be the same within the entire emission region (an input parameter of the code), and the polarisation angle is given by projection of "bulk" electron acceleration on the observer's sky. The acceleration is only determined by the curvature of magnetic field lines and the corotation of pulsar magnetosphere (no gyration component). Within the emission region of a given model, the radiation power is independent of the altitude, as the same 
number of photons is emitted per centimetre of electron's trajectory in the observer's frame. In the magnetic colatitude, the emissivity is limited to a narrow range of the footprint parameter of the magnetic field line.

\subsection{Comparison with the outer gap model}

For the outer gap model, we assumed that the emission region extends from the null-charge surface (Romani \& Yadigaroglu $1995)$ up to $r_{\max }=1.5 R_{\mathrm{lc}}$ with $\rho_{\max }=0.95 R_{\mathrm{lc}}\left(R_{\mathrm{lc}}=c P_{\mathrm{rot}} / 2 \pi\right.$ is the light cylinder radius, where $P_{\text {rot }}$ is the pulsar rotational period and $c$ is the speed of light), we considered magnetic field lines with the footprint parameter $0.75 \lesssim r_{\text {ovc }} \lesssim 0.95$, and we weighted the emission in magnetic colatitude by a Gaussian centred at $r_{\mathrm{ovc}}^{0}=0.85(\sigma=0.05)$. All the previous parameters are defined in DHR04. Different estimates exist for the Vela pulsar emission geometry, e.g. $\alpha=71^{\circ}, \zeta=65^{\circ}$ (Radhakrishnan \& Deshpande 2001), where $\alpha$ is the dipole inclination and $\zeta$ the viewing angle (see, e.g. Fig. 1 of DR03); or $\zeta=63.6^{\circ}(\mathrm{Ng} \&$ Romani 2004) from two different geometrical models of the Vela pulsar X-ray nebula; $\alpha \simeq 70^{\circ}, \zeta \simeq 61^{\circ}$ (DR03), from the twopole model of the $\gamma$-ray profile; $\alpha \sim 55^{\circ}, \zeta \sim 49^{\circ}$ (Johnston et al. 2001) from a fit to the position angle curve at 0.6 and $1.4 \mathrm{GHz}$; or $\alpha \sim 137^{\circ}, \zeta \sim 143.5^{\circ}$ (Johnston et al. 2005) from a fit at $1.4 \mathrm{GHz} ; \zeta=53.8^{\circ}$ (Krishnamohan \& Downs 1983) from a fit of the position angle curve at $2.3 \mathrm{GHz}$ and for $\alpha=60^{\circ}$. In the following, we assume $\alpha=71^{\circ}$ as a representative value.

Figure 2 shows the modelled Vela pulsar optical pulse profile and the phase-resolved polarisation properties for the representative case of $\alpha=71^{\circ}$ and $\zeta=65^{\circ}$ and for the same emission region parameters assumed above. As seen from the top panel, this set of parameters can reproduce the observed optical pulse profile of the Vela pulsar reasonably well (see, e.g. Gouiffes 1998), with two peaks separated by $\sim 0.25$ in phase. Thus, we are confident that they provide a good approximation of the Vela pulsar's emission geometry. The two lower panels show the modelled polarisation position angle $\psi$, measured from the projected pulsar rotational axis, and the depolarisation factor DP $\equiv P_{\mathrm{L}}^{\text {mod }} / P_{\mathrm{L}}^{\text {intr }}$, defined as the ratio between the modelled polarisation degree $P_{\mathrm{L}}^{\mathrm{mod}}$ at the observer's location and the intrinsic polarisation degree of the emitted radiation $P_{\mathrm{L}}^{\text {intr }}$. From our model we derived the phase-averaged value of the depolarisation factor, where the average is done over the Stokes parameters. We obtained DP $=0.68$, a typical value for many sets of model parameters. From this value we then computed the phaseaveraged, modelled polarisation degree $P_{\mathrm{L}}^{\text {mod }}$ for different values of the intrinsic polarization $P_{\mathrm{L}}^{\text {intr }}$.

Figure 3 presents the modelled phase-averaged polarisation degree $P_{\mathrm{L}}^{\mathrm{mod}}$ of the Vela pulsar as a function of the viewing angle $\zeta$ for two different values of the intrinsic optical polarisation $P_{\mathrm{L}}^{\text {intr }}$ (see caption). As seen, for $\zeta \simeq 65^{\circ}$ the modelled polarisation degree is much higher than the observed one if $P_{\mathrm{L}}^{\text {intr }}=75 \%$, while consistency would require $P_{\mathrm{L}}^{\text {intr }}$ to be as low as $13 \%$. Figure 3 also shows that for high $\alpha$ the modelled polarisation degree $P_{\mathrm{L}}^{\text {mod }}$ does not vary significantly for viewing angles $\zeta \gtrsim 40 \%$. Thus we find, in agreement with Chen et al. (1996), that the phase-averaged polarisation $P_{\mathrm{L}}$ for the outer gap models is usually more sensitive to the intrinsic properties of the emitted radiation than to the global geometric parameters $(\alpha, \zeta)$. While this makes it difficult to constrain $\alpha$ and $\zeta$ from the comparison of the modelled and observed $P_{\mathrm{L}}$, it also implies that our conclusions are less affected by the uncertain knowledge of the pulsar's emission geometry. Thus, our simulation based on the standard

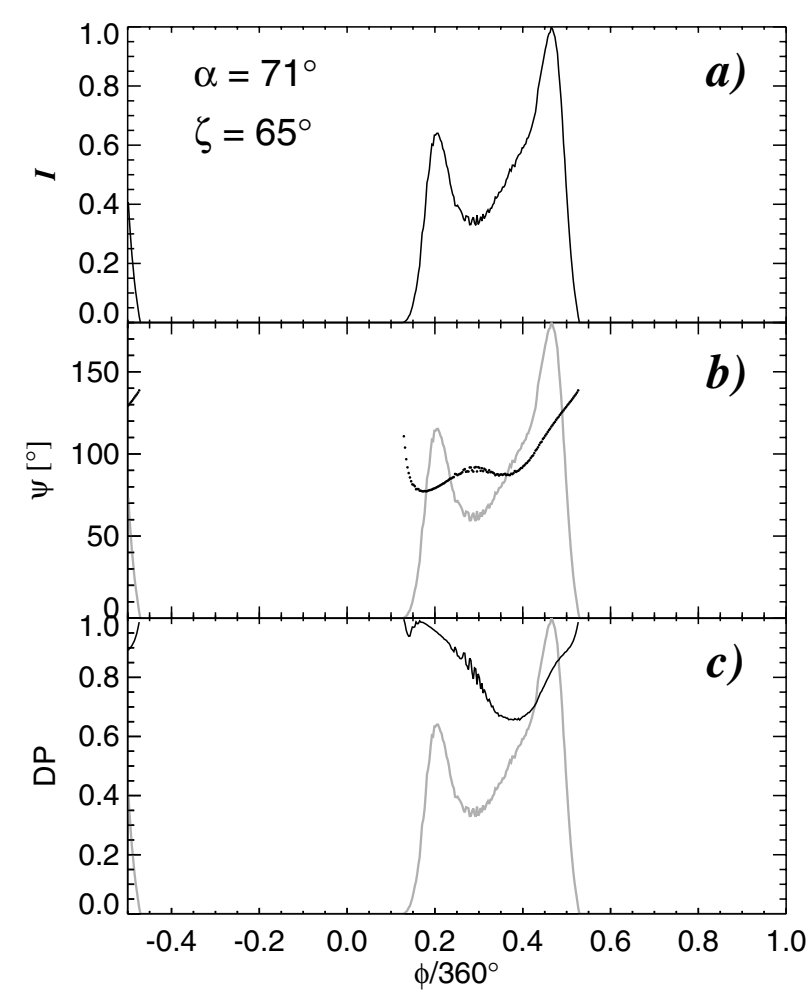

Fig. 2. Phase-resolved polarisation properties of the Vela pulsar calculated for a simplified outer gap calculation (DHR04) with the emission region parameters described in the text and assuming $\alpha=71^{\circ}$ and $\zeta=65^{\circ}$. The panels show a) the pulse profile, b) the modelled polarisation position angle $\psi, \mathbf{c}$ ) the depolarisation factor (see text for the definitions). The radio pulse is expected near phase zero. One and a half rotation period is shown. The light curve is overplotted in panels $b$ and c for reference (grey lines).

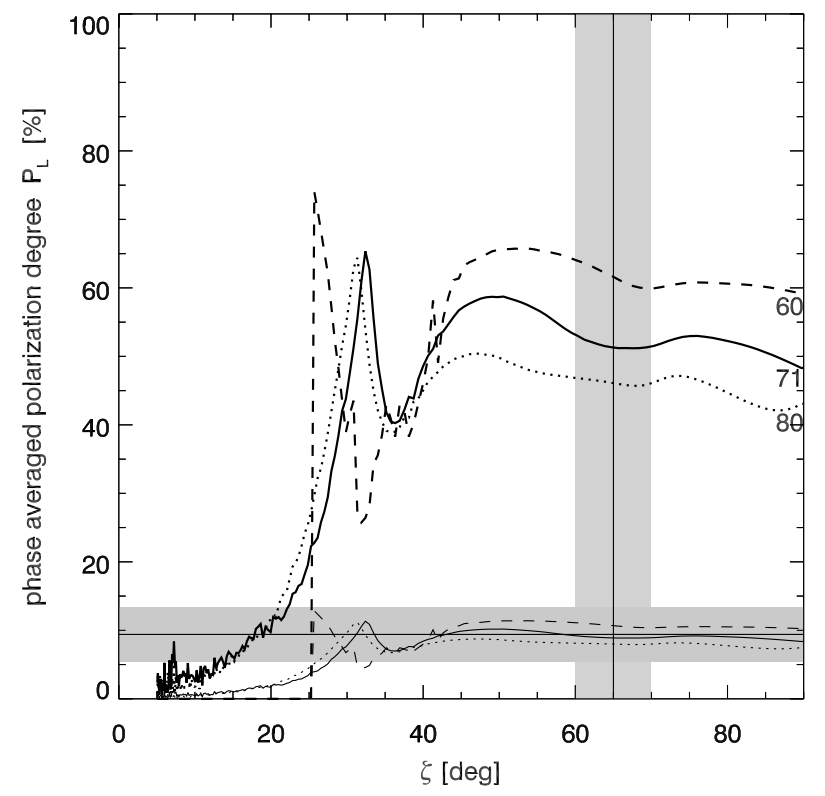

Fig. 3. Modelled phase-averaged linear polarisation degree $P_{\mathrm{L}}^{\mathrm{mod}}$ of the Vela pulsar as a function of the viewing angle $\zeta$ for a simplified outer gap calculation (DHR04) and for a dipole inclination $\alpha=71^{\circ}$ (solid). As a reference, curves for $\alpha=60^{\circ}$ (dashed) and $\alpha=80^{\circ}$ (dotted) are also shown. The thick and the thin lines correspond to an intrinsic optical polarisation $P_{\mathrm{L}}^{\text {intr }}=75 \%$ and $P_{\mathrm{L}}^{\mathrm{intr}}=13 \%$, respectively. The range of viewing angles $\zeta$ derived with various methods (see text) is marked with the vertical shaded band. The measured phase-averaged polarisation degree $P_{\mathrm{L}}$ (this work) is shown as the horizontal grey band. 


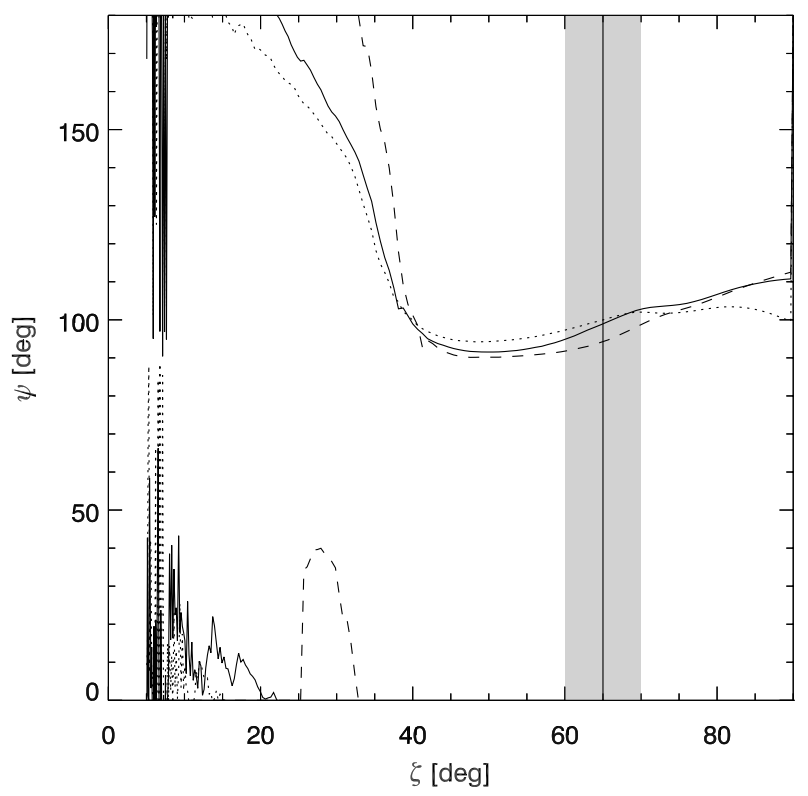

Fig. 4. Phase-averaged modelled position angle $\psi$ as a function of the viewing angle $\zeta$ for the same range of dipole inclinations $\alpha$ as in Fig. 3 . The range of viewing angles $\zeta$ derived with various methods (see text) is marked with the vertical shaded band.

outer gap model overpredicts the value of the phase-averaged polarisation, unless the intrinsic polarisation is much lower than expected. This is most likely due to the code of DHR04 not taking into account the convolution of the single electron emission beam with the structure of the emission region/magnetic field, which can be an important source of depolarisation; cf. Figs. 4b and 6c in Chen et al. (1996) and Eq. (42) in Epstein (1973).

For high $\alpha$, and for $\zeta>30^{\circ}$ the two-pole caustic model (DR03), which includes the strong emission from below the null charge surface, predicts roughly two times smaller $P_{\mathrm{L}}$ than the standard outer gap model. However, the two-pole models (including the "extended slot gap" of Muslimov \& Harding 2004) cannot reproduce the observed optical pulse shape of the Vela pulsar. This may just indicate different emission regions for the optical and for the $\gamma$-ray band, where the two-pole geometry performs quite well.

We then derived the phase-averaged, modelled polarisation position angle $\psi$, where, as before, the average is done over the Stokes parameters. Figure 4 presents the modelled phaseaveraged polarisation position angle $\psi$ as a function of the viewing angle $\zeta$ for different values of the dipole inclination $\alpha$. Only one set of curves is drawn since $\psi$ does not depend on the intrinsic polarization degree $P_{\mathrm{L}}^{\text {intr }}$. As seen, for $\zeta \geq 40^{\circ}$ the value of $\psi$ depends very little on $\alpha$ and $\zeta$. We note that the actual value of modelled position angle $\psi$ depends on which emission mechanism is actually responsible for the optical emission (e.g. synchrotron radiation or curvature radiation). In the case of Fig. $4, \psi$ was computed assuming that the emitted waves, after appropriate microscopic averaging that is not modelled, are polarized parallel to the projected bulk acceleration of electrons. This direction of polarization is characteristic of curvature radiation (Jackson 1962), and it is parallel to the projected trajectory of electrons' bulk motion (with no gyration). Instead, if the radiation is polarized perpendicular to the direction assumed in our code, as is the case for the synchrotron radiation (Rybicki $\&$ Lightman 1979), one must correct $\psi$ by subtracting $90^{\circ}$. The value of the modelled position angle should therefore be understood modulo $90^{\circ}$. For instance, for $\zeta \sim 65^{\circ}$ the modelled value of $\psi$ is either $\sim 98^{\circ}$ or $8^{\circ}$ for polarisation either parallel or perpendicular to the projected bulk acceleration, respectively. This means that the modelled phase-averaged polarisation vector is roughly perpendicular or aligned to the pulsar rotation axis. For $\zeta$ on the other hemisphere $\left(\zeta^{\prime}=180^{\circ}-\zeta\right)$, the derived position angle $\psi$ would just change sign i.e. $\psi(\zeta)=-\psi\left(\zeta^{\prime}\right)$; see Table 1 in DHR04.

A striking result visible in Fig. 4 is the proximity of $\psi$ to $\sim 90^{\circ}$ within the wide range of $\alpha$ and $\zeta$. To understand it, we recalculated the model of Fig. 2 with various kinematic effects temporarily removed from our code. We ran the code successively without the sweepback of magnetic field lines, without centrifugal acceleration, and without aberration. This exercise show that it is the last effect (the aberration of photon emission direction associated with transition from the corotating frame to the observer's frame) that is the most important in fixing $\psi$ near $\sim 90^{\circ}$. This contribution of corotational velocity to the emission direction in the observer's frame is large because the optical emission of the outer gap model originates from altitudes that are a considerable fraction of the light cylinder radius.

It is now interesting to compare the modelled polarisation position angle $\psi$ with the observed one $\theta$. However, we have to caution that a straight comparison between the two angles is not possible since the former is measured from the projected pulsar rotational axis, while the latter is measured from North. The modelled and observed polarisation position angles are thus related by the equation $\psi=\theta-\psi_{0}$, where $\psi_{0}$ is the position angle of the pulsar's projected rotational axis defined in the usual way, i.e. measured eastward from North. Thus, equating $\theta=146^{\circ} \pm 11^{\circ}$ (see Sect. 2.3) and $\psi$ allows for a direct measurement of $\psi_{0}$. For $\psi=98^{\circ} \pm 5^{\circ}$ (polarization parallel to the bulk acceleration), we obtain $\psi_{0}=48^{\circ} \pm 12^{\circ}$. Instead, for $\psi=8^{\circ} \pm 5^{\circ}$ (polarization perpendicular to bulk acceleration), we obtain $\psi_{0}=138^{\circ} \pm 12^{\circ}$. The first case implies that the pulsar projected rotation axis is almost perpendicular to the axis of symmetry of the X-ray jets and arcs, while the second case implies that it is almost aligned to them (see Sect. 2.3). If we assume that this geometry is the favoured one (e.g. Helfand et al. 2001; Pavlov et al. 2001), then $\psi_{0}=138^{\circ} \pm 12^{\circ}, \psi=8^{\circ} \pm 5^{\circ}$, and the optical radiation actually is polarised perpendicular to the bulk acceleration assumed in our code. This is consistent with a synchrotron radiation origin of the optical emission. The other way around, if we assume that the optical emission is due to synchrotron radiation, our observed polarisation position angle would imply that the pulsar rotation axis is aligned with the axis of symmetry of the X-ray jets and arcs.

Thus, our results would imply that there is a substantial alignment between the optical polarisation position angle, the pulsar rotation axis, the proper motion vector, and the axis of symmetry of the X-ray nebula. Evidence of a similar alignment has been also found from phase-resolved radio polarisation measurements of a number of radio pulsars, including Vela (Johnston et al. 2005).

\subsection{Comparison with other models}

We have qualitatively evaluated other models by comparing the predicted vs. observed polarisation properties published for the Crab pulsar, which has a very similar geometry to the Vela pulsar. The viewing angle $\zeta \simeq 60^{\circ}$ for both $\mathrm{Crab}$ and Vela $(\mathrm{Ng} \&$ Romani 2004) and the dipole inclination $\alpha$ is believed to be large in both cases (e.g. Rankin 1990). Furthermore, as shown above, the polarisation properties are found to depend rather weakly on $\alpha$ and $\zeta$. 
One of the more sophisticated models is the striped wind model (Petri \& Kirk 2005). However, this model does not account for magnetic field irregularities in the emission/reconnection region, and this is probably why the phaseaveraged polarisation degree calculated for the Crab pulsar is much larger (19-31\%, Petri \& Kirk 2005) than the observed one $^{3}$. One version of the outer gap model by Chen et al. (1996) is also notably advanced, as it includes convolution of the singleelectron emission beam with spatial distribution, electron energy distribution, and pitch angle distribution. The neglected magnetic field irregularities are probably less important in this case because the emission originates from a region well inside $R_{\mathrm{lc}}$. However, the assumed simplified geometry of the emission region (2D, no radial extent) does not seem to be adequate as it does not allow the observed pulse profile of the Crab pulsar to be reproduced. Accordingly, this model does not account for the depolarisation effect produced by the cumulation of radiation emitted from different altitudes. Using Fig. 6 in Chen et al. (1996), one can calculate a partial phase-averaged polarisation degree that is limited to the phase range within $\pm 44^{\circ}$ around the maximum of main pulse. The result $(9.4 \%)$ is two times higher than the value observed for the Crab pulsar within the same phase range ( $5.1 \%$ or $4.2 \%$ if the DC emission is excluded).

\section{Conclusions}

We have studied the phase-averaged optical polarisation properties of the Vela pulsar by reanalysing the data set used by Wagner $\&$ Seifert (2000). The fraction of linear, phase-averaged polarisation $\left(P_{\mathrm{L}}=9.4 \% \pm 4 \%\right)$ is qualitatively similar to what they found but with a larger error, which we attribute to a more realistic error analysis, thus underscoring the need for observations with a higher signal-to-noise ratio. In addition, we measured the optical linear polarisation position angle $\left(\theta=146^{\circ} \pm 11^{\circ}\right)$ for the first time, finding that it is apparently aligned with the axis of symmetry of the X-ray arcs and jets and with the pulsar's proper motion vector. We then compared our results with the expectations of the outer gap, as well as of various pulsar magnetosphere models. Interestingly, for the outer gap model we found that, for synchrotron optical emission, the observed linear polarisation position angle $\theta$ implies that the pulsar rotational axis is also aligned with the axis of symmetry of the X-ray arcs and jets. For all models we found that the computed phase-averaged polarisation degree is typically much higher than the observed one. The most probable reason for this is that these models are too idealised and do not account for some depolarisation effects. Measurements of the phase-averaged polarisation of pulsars can thus help to identify the weaknesses of various models and stimulate the development of the complex numerical codes required for calculating the expected polarisation properties. More optical polarisation observations of pulsars, now in progress with the HST, will help to provide the much-needed observational grounds for settling theoretical models.

Acknowledgements. R.P.M. warmly thanks the ESO/Chile Scientific Visitors Programme for supporting his visit at the ESO Santiago Offices where most of this work was finalised. J.D. and A.S. acknowledge the support from KBN grant 2P03D.004.24. A.S. also acknowledges support from a Deutscher Akademischer
Austausch Dienst (DAAD) fellowship. We thank the anonymous referee for his/her useful comments that contributed to improving quality of the paper.

\section{References}

Appenzeller, I., Fricke, K., Furtig, W., et al. 1998, The Messenger, 94, 1 Bagnulo, S., Boehnhardt, H., Muinonen, K., et al. 2006, A\&A, 450, 1239 Caraveo, P. A., Mereghetti, S., \& Bignami, G. F. 1994, ApJ, 423, L125

Caraveo, P. A., Mignani, R. P., DeLuca, A., \& Wagner, S. 2001a, Proc. of A decade of HST science, STScI 105, 9 [arXiv: astro-ph/0009035]

Caraveo, P. A, De Luca, A., Mignani, R. P., \& Bignami, G. F. 2001b, ApJ, 561, 930

Cheng, K. S., Ho, C., \& Ruderman, M. 1986, ApJ, 300, 500

Chen, K., Chang, H.-K., \& Ho, C. 1996, ApJ, 471, 967

Cocke, W. J., Disney, M. J., \& Taylor, D. J. 1969, Nature, 221, 525

Deshpande, A. A., \& Radhakrishnan, V. 2007, ApJ, 656, 1038

Dodson, R., Legge, D., Reynolds, J. E., \& McCulloch, P. M. 2003, ApJ, 596, 1137

Dyks, J., \& Rudak, B. 2003, ApJ, 598, 1201 (DR03)

Dyks, J., Harding, A. K., \& Rudak, B. 2004, ApJ, 606, 1125 (DHR04)

Epstein, R. I. 1973, ApJ, 183, 593

Fossati, L., Bagnulo, S., Mason, E., \& Landi Degl'Innocenti, E. 2007, Proc. of The Future of Photometric, Spectrophotometric, and Polarimetric Standardization, ed. C. Sterken, ASP Conf. Ser., in press [arXiv: astro-ph/0610637]

Gouiffes, C. 1998, Proc. of Neutron Stars and Pulsars: Thirty years after the discovery, ed. N. Shibazaki et al., Universal Academic Press, Frontiers Science Series, 24, 363

Helfand, D. J., Gotthelf, E. V., \& Halpern, J. P. 2001, ApJ, 556, 380

Jackson, J. D. 1962, Classical electrodynamics (John Wiley \& Sons, Inc., New York)

Johnston, S., van Straten, W., Kramer, M., \& Bailes, M. 2001, ApJ, 549, L101

Johnston, S., Hobbs, G., Vigeland, S., et al. 2005, MNRAS, 364, 1397

Kanbach, G., Słowikowska, A., Kellner, S., \& Steinle, H. 2005, Proc. of Astrophysical Sources of High Energy Particles and Radiation, AIP Conf. Proc., 801, 306

Kaplan, D. L. \& Moon, D. 2006, ApJ, 644, 1056

Kellner, S. 2002, Diplomarbeit, Technische Univ. München

Kern, B., Martin, C., Mazin, B., \& Halpern, J. P. 2003, ApJ, 597, 1049

Kirk, J. G., Skjaeraasen, O., \& Gallant, Y. A. 2002, A\&A, 388, L29

Krishnamohan, S., \& Downs, G. S. 1983, ApJ, 265, 372

Landi Degl'Innocenti, E., Bagnulo, S., \& Fossati, L. 2007, Proc. of The Future of Photometric, Spectrophotometric, and Polarimetric Standardization, ed. C. Sterken, ASP Conf. Ser., in press [arXiv:astro-ph/0610262]

Mignani, R. P. 2005, Proc. of The Electromagnetic Spectrum of Neutron Stars, ed. A. Baykal et al. (Springer), 210, 133

Mignani, R. P., De Luca, A., Kargaltzev, O. Y., et al. 2003, ApJ, 594, 419

Mignani, R. P., De Luca, A., \& Caraveo, P. A. 2004, Proc. of Young Neutron Stars and Their Environments, IAU Symp., 218, ed. F. Camilo, \& B. Gaensler, ASP Conf. Proc., 391

Muslimov, A. G., \& Harding, A. K. 2004, ApJ, 606, 1143

Ng, C.-Y., \& Romani, R. W. 2004, ApJ, 601, 479

Patat, F., \& Romaniello, M. 2006, PASP, 118, 146

Pavlov, G. G., Kargaltsev, O. Y., Sanwal, D., \& Garmire, G. P. 2001, ApJ, 554, L189

Petri, J., \& Kirk, J. G. 2005, ApJ, 627, L37

Radhakrishnan, V., \& Deshpande, A. A. 2001, A\&A, 379, 551

Rankin, J. M. 1990, ApJ, 352, 247

Rybicki, G. P., \& Lightman, A. P. 1979, Radiative processes in astrophysics (New York: Wiley-Interscience)

Romani, R. W., \& Yadigaroglu, I.-A. 1995, ApJ, 438, 314

Shurcliff, W. A. 1962, Polarized light (Cambridge: Harward University Press)

Smith, F. G., Jones, D. H. P., Dick, J. S. B., \& Pike, C. D. 1998, MNRAS, 233, 305

Sollerman, J., Lundqvist, P., Lindler, D., et al. 2000, ApJ, 537, 861

Wagner, S. J., \& Seifert, W. 2000, Proc. of Pulsar Astronomy - 2000 and Beyond, ed. M. Kramer et al., ASP Conf. Ser., 202, 315

Wampler, E. J., Scargle, J. D., \& Miller, J. S. 1969, ApJ, 157, L1
${ }^{3}$ In the case of the Crab pulsar, the observed phase-averaged polarisation degree is equal to $6.2 \%$. After the "DC" component is subtracted (Kellner 2002; Kanbach et al. 2005), the phase-averaged $P_{\mathrm{L}}$ becomes $3.7 \%$. 